\title{
Multi-asset Option Pricing in an Uncertain Financial Market with Jump Risk
}

\author{
Zhichao Gao ${ }^{1,2^{*}}$, Xiaosheng Wang ${ }^{2}$ and Minghu $\mathrm{Ha}^{1,2}$
}

\author{
*Correspondence: \\ zhchgao@hebeu.edu.cn \\ 1 School of Management, Hebei \\ University, Baoding 071002, China \\ ${ }^{2}$ School of Science, Hebei \\ University of Engineering, Handan \\ 056038, China
}

\begin{abstract}
Multi-asset options are created to accelerate investment among countries with the development of globalization and financial market integration. Considering the human uncertainty and the influence of sudden events such as wars and economic crisis, this paper proposes an uncertain model of multi-asset price with uncertain jumps. Option pricing formulas for the European-style dual-strike option, product option, and quotient option are derived.
\end{abstract}

Keywords: Multi-asset option, Uncertain calculus, Uncertain differential equation, Uncertain jump process

\section{Introduction}

Wiener process was used as the stock price process as early as 1900 by Bachelier. Since Wiener process allows negative value which is violating the reality, geometric Brownian motion with positive drift was proposed to describe the stock price by Samuelson [17]. In 1973, Black and Scholes [1] and Merton [16] developed the famous analytic option pricing formulas based on assuming the underlying stock price followed geometric Brownian motion. Since then, geometric Brownian motion has become the basic component part for option pricing and many studies have derived various option pricing formulas with different assumptions about the market.

When these option pricing models are dealt with, indeterminacy must be taken into account. We need a mass of data when constructing models based on probability or statistics. However, due to lack of observed data and the complexity of environment, when making decisions, people have to consult with domain experts. In this case, information and knowledge cannot be described well by random variables since human beings usually overweigh unlikely events. In order to model this type of human uncertainties, Liu [9] suggested to deal with it with uncertainty theory. Based on normality, duality, subadditivity, and product axioms, uncertainty theory was well developed in both theory aspect and practice aspect, see Liu [5-7].

Uncertain process was first introduced by Liu [14] to model the uncertain dynamic systems. Then, Liu [15] designed a canonical Liu process as the basic building block for uncertain calculus [15] and uncertain differential equation [14]. By assuming stock price followed a geometric canonical Liu process, Liu [14] derived the European option pricing

C 2016 Gao et al. Open Access This article is distributed under the terms of the Creative Commons Attribution 4.0 International License (http://creativecommons.org/licenses/by/4.0/), which permits unrestricted use, distribution, and reproduction in any medium, provided you give appropriate credit to the original author(s) and the source, provide a link to the Creative Commons license, and indicate if changes were made. 
formulas. Based on Liu's framework, American option pricing formulas for uncertain stock market were derived by Chen [2].

However, more reasonable stock price model should include jumps. Liu [14] introduced an uncertain renewal process which has uncertain event occurrence times. Yao [18] defined an uncertain integral with respect to uncertain renewal process and proposed a concept of uncertain differential equation with jumps. After that, Yu [19] proposed an uncertain stock model with positive constant jumps. Ji and Zhou [4] developed an uncertain stock model with positive and negative jumps, but the jump sizes were still constant.

In this paper, we study the uncertain jump process which consists of two parts. The continuous part of this uncertain jump process refers to an integral with respect to canonical Liu process, and the discontinuous part finitely jumps in each finite time interval. We will present the definition of integral and differential with respect to uncertain jump process. And then, we propose an uncertain model of asset price which contains multiassets and uncertain jumps. The remainder of this paper is organized as follows. The next two sections introduce some results of uncertain variable and uncertain differential equation. In the "Uncertain Jump Process" section, uncertain jump process and uncertain integral with respect to uncertain jump process are proposed. In the "Multi-asset Option Pricing Formulas" section, pricing formulas for three different exotic options with payoffs affected by at least two underlying asset prices are derived. Finally, a conclusion is drawn in the "Conclusions" section.

\section{Uncertain Variable}

Uncertainty theory is an axiomatic mathematical system founded by Liu [5] in 2007 and attracts many researchers to conduct studies on uncertain statistics [7], uncertain programming [6], uncertain risk analysis [10], uncertain finance [11], uncertain set [12], uncertain logic [13], and other application fields. To start with, some useful concepts about uncertain variables are introduced.

Let $(\Gamma, \mathcal{L}, \mathcal{M})$ be an uncertainty space where $\Gamma$ is a nonempty set, $\mathcal{L}$ is a $\sigma$-algebra defined on $\Gamma$, and $\mathcal{M}$ is an uncertain measure which was defined by Liu [5] as a set function satisfying the following axioms:

Axiom 1. (Normality axiom) $\mathcal{M}\{\Gamma\}=1$ for the $\Gamma$.

Axiom 2. (Duality axiom) $\mathcal{M}\{\Lambda\}+\mathcal{M}\left\{\Lambda^{c}\right\}=1$ for any $\Lambda \in \mathcal{L}$.

Axiom 3. (Subadditivity axiom) For every countable sequence of $\left\{\Lambda_{i}\right\} \subset \mathcal{L}$, we have

$$
\mathcal{M}\left\{\bigcup_{i=1}^{\infty} \Lambda_{i}\right\} \leq \sum_{i=1}^{\infty} \mathcal{M}\left\{\Lambda_{i}\right\} .
$$

Axiom 4. (Product axiom) Let $\left(\Gamma_{k}, \mathcal{L}_{k}, \mathcal{M}_{k}\right)$ be uncertainty spaces for $k=1,2, \cdots$ The product uncertain measure $\mathcal{M}$ is an uncertain measure satisfying

$$
\mathcal{M}\left\{\prod_{k=1}^{\infty} \Lambda_{k}\right\}=\bigwedge_{k=1}^{\infty} \mathcal{M}\left\{\Lambda_{k}\right\},
$$

where $\Lambda_{k} \in \mathcal{L}_{k}$ for $k=1,2, \cdots$, respectively. 
Following the concept of uncertainty space, uncertain variable $\xi$ is defined as a measurable function from an uncertainty space to the set of real numbers. For describing an uncertain variable $\xi$, uncertainty distribution $\Phi(x)=\mathcal{N}\{\xi \leq x\}(x \in$ $\Re, \Re$ is the set of real numbers) is proposed, and if its inverse function $\Phi^{-1}(\alpha)$ exists and is unique for each $\alpha \in(0,1), \Phi(x)$ is said to be regular.

Note that product axiom is the main difference between uncertainty theory and probability theory which implies that uncertain variable and random variable obey different operational laws.

Definition 1. (Liu [15]) The uncertain variables $\xi_{1}, \xi_{2}, \cdots, \xi_{n}$ are said to be independent if

$$
\mathcal{M}\left\{\bigcap_{i=1}^{n}\left(\xi_{i} \in B_{i}\right)\right\}=\bigwedge_{i=1}^{n} \mathcal{M}\left\{\xi_{i} \in B_{i}\right\}
$$

for any Borel sets $B_{1}, B_{2}, \cdots, B_{n}$ of real numbers.

Theorem 1. (Liu [7], operational law) Let $\xi_{1}, \xi_{2}, \ldots, \xi_{n}$ be independent uncertain variables with continuous uncertainty distributions $\Phi_{1}, \Phi_{2}, \cdots, \Phi_{n}$, respectively. If the function $f\left(x_{1}, x_{2}, \cdots, x_{n}\right)$ is strictly increasing with respect to $x_{1}, x_{2}, \cdots, x_{m}$, and strictly decreasing with respect to $x_{m+1}, x_{m+2}, \cdots, x_{n}$, then,

$$
\xi=f\left(\xi_{1}, \xi_{2}, \cdots, \xi_{n}\right)
$$

has an uncertainty distribution

$$
\Psi(x)=\sup _{f\left(x_{1}, x_{2}, \cdots, x_{n}\right)=x}\left(\min _{1 \leq i \leq m} \Phi_{i}\left(x_{i}\right) \wedge \min _{m+1 \leq i \leq n}\left(1-\Phi_{i}\left(x_{i}\right)\right)\right) .
$$

Definition 2. (Liu [5]) Let $\xi$ be an uncertain variable. Then, the expected value of $\xi$ is defined by

$$
E[\xi]=\int_{0}^{+\infty} \mathcal{M}\{\xi \geq x\} \mathrm{d} x-\int_{-\infty}^{0} \mathcal{M}\{\xi \leq x\} \mathrm{d} x .
$$

provided that at least one of the two integrals is finite.

For exploring more details and recent developments of uncertainty theory, readers can consult the book by Liu [8].

\section{Uncertain Differential Equation}

In this section, we introduce the concepts of uncertain integral and uncertain differential equation driven by canonical Liu process.

Definition 3. (Liu [15]) An uncertain process $C_{t}(t \in T, T$ is a time set) is said to be a canonical Liu process if

(i) $C_{0}=0$ and almost all sample paths are Lipschitz continuous,

(ii) $C_{t}$ has stationary and independent increments,

(iii) every increment $C_{s+t}-C_{s}$ is a normal uncertain variable with expected value 0 and variance $t^{2}$, whose uncertainty distribution is 


$$
\Phi(x)=\left(1+\exp \left(-\frac{\pi x}{\sqrt{3} t}\right)\right)^{-1}, x \in \Re
$$

Definition 4. (Liu [15]) Let $X_{t}$ be an uncertain process and let $C_{t}$ be a canonical Liu process. For any partition of closed interval $[a, b]$ with $a=t_{1}<t_{2}<\cdots<t_{k+1}=b$, the mesh is written as

$$
\Delta=\max _{1 \leq i \leq k}\left|t_{i+1}-t_{i}\right|
$$

Then, Liu integral of $X_{t}$ with respect to $C_{t}$ is defined as

$$
\int_{a}^{b} X_{t} \mathrm{~d} C_{t}=\lim _{\Delta \rightarrow 0} \sum_{i+1}^{k} X_{t_{i}} \cdot\left(C_{t_{i+1}}-C_{t_{i}}\right)
$$

provided that the limit exists almost surely and is finite. In this case, the uncertain process $X_{t}$ is said to be integrable.

Definition 5. (Liu [14]) Suppose $C_{t}$ is a canonical Liu process, and $f$ and $g$ are some given functions. Then,

$$
\mathrm{d} Z_{t}=f\left(t, Z_{t}\right) \mathrm{d} t+g\left(t, Z_{t}\right) \mathrm{d} C_{t}
$$

is called an uncertain differential equation. A solution is an uncertain process $Z_{t}$ that satisfies (8) identically in $t$.

Theorem 2. (Chen and Ralescu [3]) Suppose $C_{t}$ is a canonical Liu process, and $\mu_{t}$ and $\sigma_{t}$ are two uncertain processes. Let $Z_{t}$ be an uncertain process that satisfies the following uncertain differential equation

$$
\mathrm{d} Z_{t}=\mu_{t} \mathrm{~d} t+\sigma_{t} \mathrm{~d} C_{t}
$$

Assume $G(t, x)$ is a continuously differentiable function. Then, the uncertain process $G\left(t, Z_{t}\right)$ satisfies

$$
G\left(t, Z_{t}\right)=G\left(0, Z_{0}\right)+\int_{0}^{t}\left(\frac{\partial G}{\partial s}\left(s, Z_{s}\right)+\frac{\partial G}{\partial x}\left(s, Z_{s}\right) \cdot \mu_{s}\right) \mathrm{d} s+\int_{0}^{t} \frac{\partial G}{\partial x}\left(s, Z_{s}\right) \cdot \sigma_{s} \mathrm{~d} C_{s} .
$$

In differential form:

$$
\mathrm{d} G\left(t, Z_{t}\right)=\left(\frac{\partial G}{\partial t}\left(t, Z_{t}\right)+\frac{\partial G}{\partial x}\left(t, Z_{t}\right) \cdot \mu_{t}\right) \mathrm{d} t+\frac{\partial G}{\partial x}\left(t, Z_{t}\right) \cdot \sigma_{t} \mathrm{~d} C_{t} .
$$

There is a two-dimensional version of Theorem 2 as follows.

Theorem 3. (Chen and Ralescu [3]) Suppose $C_{t}^{(i)}(i=1,2)$ are canonical Liu processes, and $\mu_{t}^{(i)}$ and $\sigma_{t}^{(i)}(i=1,2)$ are uncertain process. Let $Z_{t}^{(i)}(i=1,2)$ be Liu processes that satisfy the following uncertain differential equations

$$
\mathrm{d} Z_{t}^{(i)}=\mu_{t}^{(i)}\left(t, Z_{t}^{(i)}\right) \mathrm{d} t+\sigma_{t}^{(i)}\left(t, Z_{t}^{(i)}\right) \mathrm{d} C_{t}^{(i)}, i=1,2 .
$$


$G\left(t, x_{1}, x_{2}\right)$ is a continuously differentiable function, then the uncertain process $G\left(t, Z_{t}^{(1)}, Z_{t}^{(2)}\right)$ satisfies

$$
\begin{aligned}
G\left(t, Z_{t}^{(1)}, Z_{t}^{(2)}\right)= & G\left(0, Z_{0}^{(1)}, Z_{0}^{(2)}\right)+\int_{0}^{t}\left(\frac{\partial G}{\partial s}+\frac{\partial G}{\partial x_{1}} \cdot \mu_{s}^{(1)}+\frac{\partial G}{\partial x_{2}} \cdot \mu_{s}^{(2)}\right) \mathrm{d} s \\
& +\int_{0}^{t}+\frac{\partial G}{\partial x_{1}} \cdot \sigma_{s}^{(1)} \mathrm{d} C_{s}^{(1)}+\int_{0}^{t} \frac{\partial G}{\partial x_{2}} \cdot \sigma_{s}^{(2)} \mathrm{d} C_{s}^{(2)}
\end{aligned}
$$

In differential form:

$$
\begin{aligned}
\mathrm{d} G\left(t, Z_{t}^{(1)}, Z_{t}^{(2)}\right)= & \left(\frac{\partial G}{\partial t}+\frac{\partial G}{\partial x_{1}} \cdot \mu_{t}^{(1)}+\frac{\partial G}{\partial x_{2}} \cdot \mu_{t}^{(2)}\right) \mathrm{d} t \\
& +\frac{\partial G}{\partial x_{1}} \cdot \sigma_{t}^{(1)} \mathrm{d} C_{t}^{(1)}+\frac{\partial G}{\partial x_{2}} \cdot \sigma_{t}^{(2)} \mathrm{d} C_{t}^{(2)}
\end{aligned}
$$

\section{Uncertain Jump Process}

In this section, we consider the jump process in $\mathrm{Ha}$ et al. (Ha, MH, Gao, ZC, Wang, XS: Managing water supply risk through an option contract in uncertain environment, submitted) as follows

$$
Z_{t}=Z_{0}+\int_{0}^{t} \mu_{s} \mathrm{~d} s+\int_{0}^{t} \sigma_{s} \mathrm{~d} C_{t}+J_{t}
$$

where $C_{t}$ is a canonical Liu process, and $\mu_{t}$ and $\sigma_{t}$ are two integrable uncertain processes. $J_{t}$ is a right-continuous pure jump uncertain process which has only finite jumps on each finite interval and is constant between jumps.

Definition 6. A process $Z_{t}$ described above is called a jump process. $Z_{t}^{c}=Z_{0}+\int_{0}^{t} \mu_{s} \mathrm{~d} s+$ $\int_{0}^{t} \sigma_{s} \mathrm{~d} C_{t}$ is called the continuous part of this process.

Definition 7. Let $Z_{t}$ be an uncertain jump process defined by (15), $X_{t}$ be an uncertain process. Then, the uncertain integral of $X_{t}$ with respect to $Z_{t}$ is defined to be

$$
\int_{0}^{t} X_{s} \mathrm{~d} Z_{s}=\int_{0}^{t} X_{s} \mu_{s} \mathrm{~d} s+\int_{0}^{t} X_{s} \sigma_{s} \mathrm{~d} C_{s}+\sum_{0<s \leq t} X_{s} \Delta J_{s},
$$

where $\Delta J_{s}$ is the jump size at time s. In differential notation:

$$
X_{t} \mathrm{~d} Z_{t}=X_{t} \mu_{t} \mathrm{~d} t+X_{t} \sigma_{t} \mathrm{~d} C_{t}+X_{t} \mathrm{~d} J_{t}=X_{t} \mathrm{~d} Z_{t}^{c}+X_{t} \mathrm{~d} J_{t} .
$$

Theorem 4. Let $Z(t)$ be a jump process and $G(t, x)$ be a continuously differentiable function. Then,

$$
\begin{aligned}
G\left(t, Z_{t}\right)= & G\left(0, Z_{0}\right)+\int_{0}^{t}\left(\frac{\partial G}{\partial s}\left(s, Z_{s}\right)+\frac{\partial G}{\partial x}\left(s, Z_{s}\right) \cdot \mu_{s}\right) \mathrm{d} s+\int_{0}^{t} \frac{\partial G}{\partial x}\left(s, Z_{s}\right) \cdot \sigma_{t} \mathrm{~d} C_{t} \\
& +\sum_{0<s \leq t}\left[G\left(s, Z_{s}\right)-G\left(s, Z_{s^{-}}\right)\right] .
\end{aligned}
$$


Proof. Let $Y_{t}=G\left(t, Z_{t}\right)$ and $T_{i}\left(i=1, \cdots, N_{t}\right)$ denote the jump times of $Z$. Let $u, v$ be in the same $\left(T_{i}, T_{i+1}\right)$. In $[u, v], Z$ evolves according to

$$
\mathrm{d} Z_{t}=\mu_{t} \mathrm{~d} t+\sigma_{t} \mathrm{~d} C_{t}
$$

By applying Theorem 2, we obtain

$$
Y_{v}-Y_{u}=\int_{u}^{v}\left(\frac{\partial G}{\partial s}\left(s, Z_{s}\right)+\frac{\partial G}{\partial x}\left(s, Z_{s}\right) \cdot \mu_{s}\right) \mathrm{d} s+\int_{u}^{v} \frac{\partial G}{\partial x}\left(s, Z_{s}\right) \cdot \sigma_{s} \mathrm{~d} C_{s} .
$$

Let $u \downarrow T_{i}$ and $v \uparrow T_{i+1}$. We have

$$
Y_{T_{i+1}}-Y_{T_{i}}=\int_{T_{i}}^{T_{i+1}^{-}}\left(\frac{\partial G}{\partial s}\left(s, Z_{s}\right)+\frac{\partial G}{\partial x}\left(s, Z_{s}\right) \cdot \mu_{s}\right) \mathrm{d} s+\int_{T_{i}}^{T_{i+1}^{-}} \frac{\partial G}{\partial x}\left(s, Z_{s}\right) \cdot \sigma_{s} \mathrm{~d} C_{s}
$$

If there is a jump at $T_{i+1}$, then the resulting change in $Y$ is $G\left(t, Z_{T_{i+1}}\right)-G\left(t, Z_{T_{i+1}^{-}}\right)$. Summing over these two contributions, we obtain

$$
\begin{aligned}
G\left(t, Z_{t}\right)= & G\left(0, Z_{0}\right)+\int_{0}^{t}\left(\frac{\partial G}{\partial s}\left(s, Z_{s}\right)+\frac{\partial G}{\partial x}\left(s, Z_{s}\right) \cdot \mu_{s}\right) \mathrm{d} s+\int_{0}^{t} \frac{\partial G}{\partial x}\left(s, Z_{s}\right) \cdot \sigma_{t} \mathrm{~d} C_{t} \\
& +\sum_{0<s \leq t}\left[G\left(s, Z_{s}\right)-G\left(s, Z_{s^{-}}\right)\right] .
\end{aligned}
$$

Hence, the theorem is proved.

Following the same method, we give the two-dimensional version of Theorem 4 .

Theorem 5. Let $Z_{t}^{(1)}$ and $Z_{t}^{(2)}$ be two jump processes and $G\left(t, x_{1}, x_{2}\right)$ be a continuously differentiable function. Then

$$
\begin{aligned}
G\left(t, Z_{t}^{(1)}, Z_{t}^{(2)}\right)= & G\left(0, Z_{0}^{(1)}, Z_{0}^{(2)}\right)+\int_{0}^{t}\left(\frac{\partial G}{\partial s}\left(s, Z_{s}^{(1)}, Z_{s}^{(2)}\right)\right. \\
& \left.+\frac{\partial G}{\partial x_{1}}\left(s, Z_{s}^{(1)}, Z_{s}^{(2)}\right) \cdot \mu_{s}^{(1)}+\frac{\partial G}{\partial x_{2}}\left(s, Z_{s}^{(1)}, Z_{s}^{(2)}\right) \cdot \mu_{s}^{(2)}\right) \mathrm{d} s \\
& +\int_{0}^{t} \frac{\partial G}{\partial x_{1}}\left(s, Z_{s}^{(1)}, Z_{s}^{(2)}\right) \cdot \sigma_{t}^{(1)} \mathrm{d} C_{t}^{(1)}+\int_{0}^{t} \frac{\partial G}{\partial x_{2}}\left(s, Z_{s}^{(1)}, Z_{s}^{(2)}\right) \cdot \sigma_{t}^{(2)} \mathrm{d} C_{t}^{(2)} \\
& +\sum_{0<s \leq t}\left[G\left(s, Z_{s}^{(1)}, Z_{s}^{(2)}\right)-G\left(s, Z_{s^{-}}^{(1)}, Z_{s^{-}}^{(2)}\right)\right] .
\end{aligned}
$$

\section{Multi-asset Option Pricing Formulas}

With the development of globalization and cross-market integration, multi-asset options are created. In this section, we suppose that the asset price follows uncertain jump processes whose right-continuous pure jump part is determined by an uncertain renewal reward process. Let $r$ denote the risk-free interest rate, $e$ be the log drift, $\sigma$ be the log diffusion, and $C_{t}$ be a canonical Liu process. $R_{t}=\sum_{i=1}^{N_{t}} Y_{i}$ is an uncertain renewal reward process where $N_{t}$ is the number of renewals in $(0, t]$ with independent and identically distributed (i.i.d) uncertain interarrival times $X_{1}, X_{2}, \cdots$ and $Y_{1}, Y_{2}, \cdots$ are i.i.d uncertain variables. We assume that $X_{1}, Y_{1}, X_{2}, Y_{2}, \cdots$ are also independent. Then, the underlying uncertain model of asset price is given by

$$
\mathrm{d} Z_{t}=e Z_{t} \mathrm{~d} t+\sigma Z_{t} \mathrm{~d} C_{t}+Z_{t-} \mathrm{d} R_{t}
$$


Let $Y_{i}>-1, i=1,2, \cdots$. This assumption guarantees that the asset price can jump down but it cannot jump from positive to negative or to zero. The solution to (20) is

$$
Z_{t}=Z_{0} \exp \left\{e t+\sigma C_{t}\right\} \prod_{i=1}^{N_{t}}\left(1+Y_{i}\right)
$$

To check if (21) satisfies the uncertain differential Eq. (20), we define the continuous part

$$
Z_{t}^{c}=Z_{0} \exp \left\{e t+\sigma C_{t}\right\}
$$

and the pure jump part

$$
J_{t}=\prod_{i=1}^{N_{t}}\left(1+Y_{i}\right)
$$

Then, $Z_{t}=Z_{t}^{c} J_{t}$. By using Theorem 2, we have

$$
\mathrm{d} Z_{t}^{c}=e Z_{t}^{c} \mathrm{~d} t+\sigma Z_{t}^{c} \mathrm{~d} C_{t}
$$

And the jump size of $J_{t}$ at time $t$ is

$$
\Delta J_{t}=J_{t}-J_{t-}=J_{t-}\left(1+Y_{i}\right)-J_{t}=J_{t-} \Delta R_{t} .
$$

By using Theorem 5 and the above equations, we obtain

$$
\begin{aligned}
Z_{t} & =Z_{t}^{c} J_{t} \\
& =Z_{0}^{c} J_{0}+\int_{0}^{t} e Z_{s}^{c} J_{s} \mathrm{~d} s+\int_{0}^{t} \sigma Z_{s}^{c} J_{s} \mathrm{~d} C_{s}+\sum_{0<s \leq t}\left[Z_{s}^{c} J_{s}-Z_{s-}^{c} J_{s-}\right] \\
& =Z_{0}^{c} J_{0}+\int_{0}^{t} e Z_{s}^{c} J_{s} \mathrm{~d} s+\int_{0}^{t} \sigma Z_{s}^{c} J_{s} \mathrm{~d} C_{s}+\sum_{0<s \leq t} Z_{s-}^{c} J_{s-} \Delta R_{s} \\
& =Z_{0}+\int_{0}^{t} e Z_{s} \mathrm{~d} s+\int_{0}^{t} \sigma Z_{s} \mathrm{~d} C_{s}+\sum_{0<s \leq t} Z_{s-} \Delta R_{s} .
\end{aligned}
$$

This verifies that (21) is the solution to (20).

Theorem 6. Assume $C_{t}$ has distribution $\Phi_{t}(x), Y_{1}, Y_{2}, \cdots$ and $X_{1}, X_{2}, \cdots$ have distributions $F(x)$ and $H(x)$, respectively. Then, $Z_{t}$ has an uncertainty distribution

$$
\begin{aligned}
\Psi_{t}(x)= & \sup _{x_{1} x_{2}=x / Z_{0}}\left\{\Phi_{t}\left(\frac{\ln x_{1}-e t}{\sigma}\right)\right. \\
& \left.\wedge\left(\max _{k \geq 0}\left(1-H\left(\frac{t}{k+1}\right)\right) \wedge F\left(\exp \left(\frac{x_{2}}{k}\right)-1\right)\right)\right\} .
\end{aligned}
$$


Proof. Because $Y_{i}$ has distribution $F(x), \ln \left(1+Y_{i}\right)$ has distribution $F(\exp (x)-1)$. $\prod_{i=1}^{N_{t}}\left(1+Y_{i}\right)$ has an uncertainty distribution

$$
\begin{aligned}
\Upsilon_{t}(x) & =\mathcal{M}\left\{\prod_{i=1}^{N_{t}}\left(1+Y_{i} \leq x\right\}\right. \\
& =\mathcal{M}\left\{\sum_{i=1}^{N_{t}} \ln \left(1+Y_{i}\right) \leq \ln x\right\} \\
& =\mathcal{M}\left\{\bigcup_{k=0}^{\infty}\left(N_{t}=k\right) \cap \sum_{i=1}^{k} \ln \left(1+Y_{i}\right) \leq x\right\} \\
& =\mathcal{M}\left\{\bigcup_{k=0}^{\infty}\left(N_{t}=k\right) \cap\left(\ln \left(1+Y_{1}\right) \leq \frac{x}{k}\right)\right\} \\
& =\max _{k \geq 0} \mathcal{M}\left\{\left(N_{t} \leq k\right) \cap\left(\ln \left(1+Y_{1}\right) \leq \frac{x}{k}\right)\right\} \\
& =\max _{k \geq 0} \mathcal{M}\left\{\left(N_{t} \leq k\right)\right\} \wedge \mathcal{M}\left\{\left(\ln \left(1+Y_{1}\right) \leq \frac{x}{k}\right)\right\} \\
& =\max _{k \geq 0}\left(1-H\left(\frac{t}{k+1}\right)\right) \wedge F\left(\exp \left(\frac{x}{k}\right)-1\right)
\end{aligned}
$$

On the other hand, $\exp \left\{e t+\sigma C_{t}\right\}$ has uncertainty distribution $\Phi\left(\frac{\ln x-e t}{\sigma}\right)$. By the operational law of uncertain variables, $Z_{t}$ has an uncertainty distribution

$$
\begin{aligned}
\Psi_{t}(x)= & \mathcal{M}\left\{Z_{t} \leq x\right\} \\
= & \mathcal{M}\left\{Z_{0} \exp \left\{e t+\sigma C_{t}\right\} \prod_{i=1}^{N_{t}}\left(1+Y_{i} \leq x\right\}\right. \\
= & \sup _{x_{1} x_{2}=x / Z_{0}} \mathcal{M}\left\{\left(\exp \left\{e t+\sigma C_{t}\right\} \leq x_{1}\right) \cap\left(\prod_{i=1}^{N_{t}}\left(1+Y_{i}\right) \leq x_{2}\right)\right\} \\
= & \sup _{x_{1} x_{2}=x / Z_{0}} \mathcal{M}\left\{\left(\exp \left\{e t+\sigma C_{t}\right\} \leq x_{1}\right)\right\} \wedge \mathcal{M}\left\{\left(\prod_{i=1}^{N_{t}}\left(1+Y_{i}\right) \leq x_{2}\right)\right\} \\
= & \sup _{x_{1} x_{2}=x / Z_{0}}\left\{\Phi _ { t } ( \frac { \operatorname { l n } x _ { 1 } - e t } { \sigma } ) \wedge \left(\max _{k \geq 0}\left(1-H\left(\frac{t}{k+1}\right)\right)\right.\right. \\
& \left.\left.\wedge F\left(\exp \left(\frac{x_{2}}{k}\right)-1\right)\right)\right\} .
\end{aligned}
$$

The theorem is proved.

\section{Dual-Strike Options}

A dual-strike option is an option which has two strike prices written on two underlying financial assets. In an uncertain environment, suppose the two assets $Z_{t}^{(1)}$ and $Z_{t}^{(2)}$ both follow the uncertain jump process given by (20) and are independent. Then, the payoff of a European dual-strike option is $\max \left\{\omega_{1}\left(Z_{1}^{(1)}-K_{1}\right), \omega_{2}\left(Z_{1}^{(2)}-K_{2}\right), 0\right\}$ where $K_{1}$ and $K_{2}$ are the strike prices, $\omega_{1}$ and $\omega_{2}$ are 1 for a call option and -1 for a put option. By discounting the expected payoff at expiration date $T$, the price of the dual-strike option is

$$
f_{d s}=\exp (-r T) E\left[\max \left\{\omega_{1}\left(Z_{t}^{(1)}-K_{1}\right), \omega_{2}\left(Z_{t}^{(2)}-K_{2}\right), 0\right\}\right] .
$$

In the following, we derive the pricing formula for dual-strike option with $\omega_{1}=\omega_{2}=1$. 
Theorem 7. The price of a European dual-strike option is

$$
f_{d s}=\int_{0}^{+\infty}\left(1-\Psi_{T}^{(1)}\left(x+K_{1}\right)\right) \vee\left(1-\Psi_{T}^{(2)}\left(x+K_{2}\right)\right) \mathrm{d} x
$$

where

$$
\begin{aligned}
\Psi_{T}^{(i)}(x)= & \sup _{x_{1} x_{2}=x / Z_{0}}\left\{\Phi _ { T } ( \frac { \operatorname { l n } x _ { 1 } - e _ { i } t } { \sigma _ { i } } ) \wedge \left(\max _{k \geq 0}\left(1-H^{(i)}\left(\frac{t}{k+1}\right)\right)\right.\right. \\
& \left.\left.\wedge F^{(i)}\left(\exp \left(\frac{x_{2}}{k}\right)-1\right)\right)\right\}, i=1,2 .
\end{aligned}
$$

Proof. By the definition of expected value

$$
\begin{aligned}
& E\left[\max \left\{\left(Z_{T}^{(1)}-K_{1}\right),\left(Z_{T}^{(2)}-K_{2}\right), 0\right\}\right] \\
& =\int_{0}^{+\infty} \mathcal{M}\left\{\left(Z_{T}^{(1)}-K_{1} \geq x\right) \cup\left(Z_{T}^{(2)}-K_{2} \geq x\right)\right\} \mathrm{d} x \\
& =\int_{0}^{+\infty} \mathcal{M}\left\{Z_{T}^{(1)} \geq x+K_{1}\right\} \vee \mathcal{M}\left\{Z_{T}^{(2)} \geq x+K_{2}\right\} \mathrm{d} x \\
& =\int_{0}^{+\infty}\left(1-\mathcal{M}\left\{Z_{T}^{(1)}<x+K_{1}\right\}\right) \vee\left(1-\mathcal{M}\left\{Z_{T}^{(2)}<x+K_{2}\right\}\right) \mathrm{d} x \\
& =\int_{0}^{+\infty}\left(1-\Psi_{T}^{(1)}\left(x+K_{1}\right)\right) \vee\left(1-\Psi_{T}^{(2)}\left(x+K_{2}\right)\right) \mathrm{d} x .
\end{aligned}
$$

Using (27), we know that

$$
\begin{aligned}
\Psi_{T}^{(i)}(x)= & \sup _{x_{1} x_{2}=x / Z_{0}}\left\{\Phi _ { T } ( \frac { \operatorname { l n } x _ { 1 } - e _ { i } t } { \sigma _ { i } } ) \wedge \left(\max _{k \geq 0}\left(1-H^{(i)}\left(\frac{t}{k+1}\right)\right)\right.\right. \\
& \left.\left.\wedge F^{(i)}\left(\exp \left(\frac{x_{2}}{k}\right)-1\right)\right)\right\}, i=1,2 .
\end{aligned}
$$

Hence, the deal-strike option pricing formula is verified.

\section{Product Options}

A product option is an option which is written on the product of two financial assets. Let $Z_{t}^{(1)}$ and $Z_{t}^{(2)}$ be uncertain jump processes which satisfy (20) and (1). Then, the price of a European-style product option with strike price $K$ and expiration date $T$ is

$$
f_{p}=\exp (-r T) E\left[\max \left\{\omega Z_{T}^{(1)} Z_{T}^{(2)}-\omega K, 0\right\}\right],
$$

where $\omega$ is 1 for a call option and -1 for a put option. In the following, we consider the product call option.

Theorem 8. The price of a European product option is

$$
f_{p}=\int_{0}^{+\infty}\left(1-\Psi_{p}(x+K)\right) \mathrm{d} x
$$

where

$$
\begin{gathered}
\Psi_{p}(x)=\sup _{x_{1} x_{2}=x} \Psi_{T}^{(1)}\left(x_{1}\right) \wedge \Psi_{T}^{(2)}\left(x_{2}\right), \\
\Psi_{T}^{(i)}(x)=\sup _{x_{1} x_{2}=x / Z_{0}}\left\{\Phi _ { T } ( \frac { \operatorname { l n } x _ { 1 } - e _ { i } t } { \sigma _ { i } } ) \wedge \left(\max _{k \geq 0}\left(1-H^{(i)}\left(\frac{t}{k+1}\right)\right)\right.\right. \\
\left.\left.\wedge F^{(i)}\left(\exp \left(\frac{x_{2}}{k}\right)-1\right)\right)\right\}, i=1,2 .
\end{gathered}
$$


Proof. By the operational law, we can obtain the distribution of $Z_{T}^{(1)} Z_{T}^{(2)}$

$$
\Psi_{p}(x)=\sup _{x_{1} x_{2}=x} \Psi_{T}^{(1)}\left(x_{1}\right) \wedge \Psi_{T}^{(2)}\left(x_{2}\right)
$$

where $\Psi_{T}^{(1)}$ and $\Psi_{T}^{(2)}$ are distribution functions of $Z_{T}^{(1)}$ and $Z_{T}^{(2)}$. Then, by the definition of expected value,

$$
\begin{aligned}
E\left[\max \left\{Z_{T}^{(1)} Z_{T}^{(2)}-K, 0\right\}\right] & =\int_{0}^{+\infty} \mathcal{M}\left\{\max \left\{Z_{T}^{(1)} Z_{T}^{(2)}-K, 0\right\} \geq x\right\} \mathrm{d} x \\
& =\int_{0}^{+\infty} \mathcal{M}\left\{Z_{T}^{(1)} Z_{T}^{(2)} \geq x+K\right\} \mathrm{d} x \\
& =\int_{0}^{+\infty}\left(1-\mathcal{M}\left\{Z_{T}^{(1)} Z_{T}^{(2)}<x+K\right\}\right) \mathrm{d} x \\
& =\int_{0}^{+\infty}\left(1-\Psi_{p}(x+K)\right) \mathrm{d} x .
\end{aligned}
$$

Thus, the product option pricing formula is verified.

\section{Quotient Options}

A quotient option is an option which is written on the ratio of two financial assets. Let $Z_{t}^{(1)}$ and $Z_{t}^{(2)}$ be uncertain jump processes which satisfy (20) and (1). Then, the price of a European-style quotient option with strike price $K$ and expiration date $T$ is

$$
f_{q}=\exp (-r T) E\left[\max \left\{\omega \frac{Z_{T}^{(1)}}{Z_{T}^{(2)}}-\omega K, 0\right\}\right]
$$

where $\omega$ is 1 for a call option and -1 for a put option. In the following, we derive the pricing formula for quotient call option.

Theorem 9. The price of a European quotient option is

$$
f_{q}=\int_{0}^{+\infty}\left(1-\Psi_{q}(x+K)\right) \mathrm{d} x
$$

where

$$
\begin{gathered}
\Psi_{q}(x)=\sup _{y>0} \Psi_{T}^{(1)}(x y) \wedge\left(1-\Psi_{T}^{(2)}(y)\right), \\
\Psi_{T}^{(i)}(x)=\sup _{x_{1} x_{2}=x / Z_{0}}\left\{\Phi _ { T } ( \frac { \operatorname { l n } x _ { 1 } - e _ { i } t } { \sigma _ { i } } ) \wedge \left(\max _{k \geq 0}\left(1-H^{(i)}\left(\frac{t}{k+1}\right)\right)\right.\right. \\
\left.\left.\wedge F^{(i)}\left(\exp \left(\frac{x_{2}}{k}\right)-1\right)\right)\right\}, i=1,2 .
\end{gathered}
$$

Proof. By the operational law, we can obtain the distribution of $Z_{T}^{(1)} / Z_{T}^{(2)}$,

$$
\Psi_{q}(x)=\sup _{y>0} \Psi_{T}^{(1)}(x y) \wedge\left(1-\Psi_{T}^{(2)}(y)\right)
$$


where $\Psi_{T}^{(1)}$ and $\Psi_{T}^{(2)}$ are distribution functions of $Z_{T}^{(1)}$ and $Z_{T}^{(2)}$. Then, by the definition of expected value,

$$
\begin{aligned}
E\left[\max \left\{\frac{Z_{T}^{(1)}}{Z_{T}^{(2)}}-K, 0\right\}\right] & =\int_{0}^{+\infty} \mathcal{M}\left\{\max \left\{\frac{Z_{T}^{(1)}}{Z_{T}^{(2)}}-K, 0\right\} \geq x\right\} \mathrm{d} x \\
& =\int_{0}^{+\infty} \mathcal{M}\left\{\frac{Z_{T}^{(1)}}{Z_{T}^{(2)}} \geq x+K\right\} \mathrm{d} x \\
& =\int_{0}^{+\infty}\left(1-\mathcal{M}\left\{\frac{Z_{T}^{(1)}}{Z_{T}^{(2)}}<x+K\right\}\right) \mathrm{d} x \\
& =\int_{0}^{+\infty}\left(1-\Psi_{q}(x+K)\right) \mathrm{d} x .
\end{aligned}
$$

The quotient option pricing formula is verified.

\section{Conclusions}

More realistic stock model should consider jumps in the price process. This paper proposed an uncertain jump process and an uncertain model of multi-asset price with jumps. Option pricing formulas for European-style dual-strike option, product option, and quotient option were derived. In the future, various other multi-asset options in uncertain environment can be studied.

\section{Competing interests}

The authors declare that they have no competing interests.

\section{Acknowledgements}

This work was supported by the National Natural Science Foundation of China (No. 61073121), Natural Science Foundation of Hebei Province (Nos. F2012402037 and G2013402063), and Social Science Foundation of Hebei Province (No. HB15GL119).

Received: 20 October 2015 Accepted: 15 December 2015

Published online: 07 January 2016

\section{References}

1. Black, F, Scholes, M: The pricing of options and corporate liabilities. J. Polit. Econ. 81(3), $637-654$ (1973)

2. Chen, XW: American option pricing formula for uncertain financial market. Int. J. Oper. Res. 8(2), 32-37 (2011)

3. Chen, XW, Ralescu, DA: Liu process and uncertain calculus. J. Uncertainty Anal. Appl. 1, Article, 3 (2013)

4. Ji, X, Zhou, J: Option pricing for an uncertain stock model with jumps. Soft Comput. 19(11), 3323-3329 (2015)

5. Liu, BD: Uncertainty theory. 2nd ed. Springer-Verlag, Berlin (2007)

6. Liu, BD: Theory and practice of uncertain programming. 2nd ed. Springer-Verlag, Berlin (2009)

7. Liu, BD: Uncertainty theory: a branch of mathematics for modeling human uncertainty. Springer-Verlag, Berlin (2011)

8. Liu, BD: Uncertainty theory. 5th ed (2015). http://orsc.edu.cn/liu/ut.pdf

9. Liu, BD: Why is there a need for uncertainty theory. J. Uncertain Syst. 6(1), 3-10 (2012)

10. Liu, BD: Uncertain risk analysis and uncertain reliability analysis. J. Uncertain Syst. 4(3), 163-170 (2010)

11. Liu, BD: Toward uncertain finance theory. J. Uncertainty Anal. Appl. 1, Article, 1 (2013)

12. Liu, BD: Uncertain set theory and uncertain inference rule with application to uncertain control. J. Uncertain Syst. 4(2), 83-98 (2010)

13. Liu, BD: Uncertain logic for modeling human language. J. Uncertain Syst. 5(1), 3-20 (2011)

14. Liu, BD: Fuzzy process, hybrid process and uncertain process. J. Uncertain Syst. 2(1), 3-16 (2008)

15. Liu, BD: Some research problems in uncertainty theory. J. Uncertain Syst. 3(1), 3-10 (2009)

16. Merton, RC: Theory of rational option pricing. Bell J. Econ. Manag. Sci. 4(1), 141-183 (1973)

17. Samuelson, PA: Rational theory of warrant pricing. Ind. Manag. Rev. 6(2), 13-32 (1965)

18. Yao, K: Uncertain calculus with renewal process. Fuzzy Optim Decis. Making. 11(3), 285-297 (2012)

19. Yu, XC: A stock model with jumps for uncertain markets. Int. J. Uncertainty, Fuzziness Knowledge-Based Syst. 20(3), $421-432(2012)$ 